\title{
Sequential Warped Products and Their Applications
}

\author{
Sinem Güler* \\ (Communicated by Uday Chand De)
}

\begin{abstract}
In this paper, we study the sequential warped product manifolds, which are the natural generalizations of singly warped products. Many spacetime models that characterize the universe and the solutions of Einstein's field equations are known to have this new structure. For this reason, first, we investigate the geometry of sequential warped product manifold under some conditions of concircular curvature tensor. We also study the conformal and gradient almost Ricci solitons on the sequential warped product. These conditions allow us to obtain some interesting expressions for the Riemann curvature and the Ricci tensors of its base and fiber from the geometrical and the physical point of view. Then, we give two important applications of this concept in the Lorentzian settings, which are sequential generalized Robertson-Walker spacetimes and sequential standard static spacetimes and obtain the form of the warping functions. Also, by considering generalized quasi Einsteinian conditions on these spacetimes, we find some specific formulas for the Ricci tensors of the bases and fibers. Finally, we end this work with some examples for this structure.
\end{abstract}

Keywords: Sequential warped product, concircular curvature tensor, generalized Robertson-Walker spacetime, standard static spacetime, conformal soliton, generalized quasi Einstein manifold.

AMS Subject Classification (2020): Primary: 53C21 ; Secondary: 53C25; 53C50; 53C80.

\section{Introduction}

In 1969, Bishop and O'Neill [5] have defined the warped product of two Riemannian manifolds $\left(B, g_{B}\right)$ and $\left(F, g_{F}\right)$ as the product $B \times F$ equipped with the metric $g_{B} \oplus f^{2} g_{F}$ and denoted by $B \times{ }_{f} F$, where the smooth function $f: B \rightarrow(0, \infty)$ is called the warping function. Then in 1983, warped products of semi-Riemannian (not necessarily Lorentzian) manifolds and their Riemannian and Ricci curvature tensors were given in [19]. Then it turned out that the standard spacetime models of the universe and many other fundamental examples of relativistic spacetimes that are the solutions of Einstein's field equations are such warped products. From this reason, warped products play very important roles in differential geometry as well as in general relativity. Two very well-known examples of this notion are four dimensional Schwarzchild and de Sitter solutions of Einstein field equations.

Let $\mathbb{R}^{4}$ be given by the coordinates $(t, r, \theta, \phi)$, where $(r, \theta, \phi)$ are the usual spherical coordinates of $\mathbb{R}^{3}$. For a positive constant $m$, the exterior Schwarzchild spacetime is defined on the subset $r>2 m$ of $\mathbb{R}^{4}$ and for this region the Schwarzchild metric is given by

$$
\mathrm{d} s^{2}=-\left(1-\frac{2 m}{r}\right) \mathrm{d} t^{2}+\left(1-\frac{2 m}{r}\right)^{-1} \mathrm{~d} r^{2}+r^{2}\left(\mathrm{~d} \theta^{2}+\sin ^{2} \theta \mathrm{d} \phi^{2}\right) .
$$

Thus, if $S^{2}$ denotes the unit shpere, the exterior Schwarzchild spacetime is the Lorentzian warped product $N=P_{1} \times_{r} S^{2}$, where $P_{1}=\left\{(t, r) \in \mathbb{R} \times \mathbb{R}^{+}: r>2 m\right\}$. 
The de Sitter spacetime of constant positive sectional curvature $\frac{1}{r^{2}}$ covered by the global coordinates $(t, \alpha, \theta, \phi)$ with $-\infty<t<\infty, 0 \leq \alpha, \theta \leq \pi$ and $0 \leq \phi \leq 2 \pi$ is given by

$$
\mathrm{d} s^{2}=-\mathrm{d} t^{2}+r^{2} \cosh ^{2}\left(\frac{t}{r}\right)\left[\mathrm{d} \alpha^{2}+\sin ^{2} \alpha\left(\mathrm{d} \theta^{2}+\sin ^{2} \theta \mathrm{d} \phi^{2}\right)\right] .
$$

Thus, this also can be considered as the Lorentzian warped product $\mathbb{S}_{1}^{n}=\mathbb{R} \times_{f} S^{3}$, where $S^{3}$ denotes the complete Riemannian manifold of constant sectional curvature and $f(t)=r \cosh \left(\frac{t}{r}\right)$ is the warping function. Other examples apart from these may be listed as black hole, Kerr, Reissner-Nordström and the anti-de Sitter metrics. As can be easily seen from the equations (1.1) and (1.2), in many warped product manifolds, their bases, fibers or both of them can also have the warped product structure, [22]. Moreover, in string theory, for the Calabi-Yau manifold (i.e. Ricci flat Riemannian manifold admitting a complex structure) $F$ and the de Sitter spacetime $\mathbb{S}_{1}^{n}$, the warped product $\mathbb{S}_{1}^{n} \times F$ has the same feature, [4]. Thus, a new concept of warped product metric has been introduced in which its base or fiber or both also have the warped product structure and it is called sequential warped product, [20,11]. The formal definition of this notion is given as follows:

Definition 1.1. Let $\left(M_{i}, g_{i}\right)$ be three semi-Riemannian manifolds of dimensions $m_{i}(i=1,2,3)$ respectively, $f: M_{1} \rightarrow(0, \infty)$ and $h: M_{1} \times M_{2} \rightarrow(0, \infty)$ be two smooth functions. Then the sequential warped product is the product manifold $\bar{M}=\left(M_{1} \times_{f} M_{2}\right) \times_{h} M_{3}$ endowed with the metric

$$
\bar{g}=\left(g_{1} \oplus f^{2} g_{2}\right) \oplus h^{2} g_{3} .
$$

Both $f$ and $h$ are called the warping functions.

Notation 1. (i) Throughout the paper, we will use Einstein's summation convention. All the objects will be assumed to be smooth and all the manifolds are connected.

(ii) All objects having "bar" symbol represent the objects of the sequential warped product manifold and all objects having the indices or powers $i$ denote the objects of the manifold $\left(M_{i}, g_{i}\right)$, where $i=1,2,3$.

(iii) The Riemannian curvature tensor is defined by $R(X, Y) Z=\left[\nabla_{X}, \nabla_{Y}\right] Z-\nabla_{[X, Y]} Z$, the Ricci tensor is $\operatorname{Ric}(X, Y)=\sum_{i=1}^{n} R\left(e_{i}, X, Y, e_{i}\right)$ and the scalar curvature $r=\sum_{i=1}^{n} \operatorname{Ric}\left(e_{i}, e_{i}\right)$, where $\left\{e_{i}: i=1, \ldots, n\right\}$ denotes orthonormal basis over the manifold.

(iv) For any $X, Y \in \chi(M)$, the Hessian of a smooth function $\phi$ is the second order covariant differentiation defined by $H^{\phi}(X, Y)=X Y(\phi)-\left(\nabla_{X} Y\right) \phi=g\left(\nabla_{X} \operatorname{grad} \phi, Y\right)$.

(v) On a sequential warped product $\bar{M}=\left(M_{1} \times_{f} M_{2}\right) \times_{h} M_{3}$, every vector field $X$ can be decomposed as the sum

$$
X=X_{1}+X_{2}+X_{3}, \text { where } X_{i} \in \chi\left(M_{i}\right), \quad i=1,2,3 .
$$

This paper is organized as follows: After Introduction, first in Section 2 we give detailed preliminaries about the geometry of sequential warped product manifolds and the concircular curvature tensor. Section 3 is devoted to the geometry of concircular curvature tensor and some gradient solitons on sequential warped product manifolds. In Subsection 3.1, we give description of the concircular curvature on sequential warped product and obtain necessary and sufficient conditions for concircular flatness. We also study the conformal and gradient almost Ricci solitons on the sequential warped product. In Subsection 3.2, we investigate the concircular symmetry and in Subsection 3.3, we analyse the harmonicity of concircular curvature on sequential warped product. In Section 4, we study two important applications of this concept in the Lorentzian setting, which are sequential generalized Robertson-Walker spacetimes and sequential standard static spacetimes. In this way, we are able to obtain the form of the warping functions in some cases of sequential warped product spacetimes. Also, by considering the generalized quasi Einsteinian condition on these spacetimes, we find some specific formulas for the Ricci tensors of its base and fiber. Finally, we construct two examples for sequential generalized Robertson-Walker spacetimes, which are also generalized quasi Einstein manifolds.

\section{Preliminaries}

\subsection{Sequential Warped Products}

In this section, we give the basic formulas for the Levi-Civita connection, Riemannian, Ricci and the scalar curvature of the sequential warped products that will be used in the proofs of our main theorems. We skip the proofs that are long but straightforward, as is the case of warped product manifolds. 
Let $\bar{M}=\left(M_{1} \times_{f} M_{2}\right) \times_{h} M_{3}$ be a sequential warped product endowed with the metric $\bar{g}=\left(g_{1} \oplus f^{2} g_{2}\right) \oplus h^{2} g_{3}$ and let $X_{i}, Y_{i}, Z_{i} \in \chi\left(M_{i}\right)$, for $i=1,2,3$. Then, we have:

Lemma 2.1. [11] The components of the Levi-Civita connection on $(\bar{M}, \bar{g})$ are given by:

1. $\bar{\nabla}_{X_{1}} Y_{1}=\nabla_{X_{1}}^{1} Y_{1}$

2. $\bar{\nabla}_{X_{1}} X_{2}=\bar{\nabla}_{X_{2}} X_{1}=X_{1}(\ln f) X_{2}$,

3. $\bar{\nabla}_{X_{2}} Y_{2}=\nabla_{X_{2}}^{2} Y_{2}-f g_{2}\left(X_{2}, Y_{2}\right) \operatorname{grad}_{1} f$,

4. $\bar{\nabla}_{X_{3}} X_{1}=\bar{\nabla}_{X_{1}} X_{3}=X_{1}(\ln h) X_{3}$

5. $\bar{\nabla}_{X_{2}} X_{3}=\bar{\nabla}_{X_{3}} X_{2}=X_{2}(\ln h) X_{3}$

6. $\bar{\nabla}_{X_{3}} Y_{3}=\nabla_{X_{3}}^{3} Y_{3}-h g_{3}\left(X_{3}, Y_{3}\right)$ gradh.

Note that, $H_{1}^{f}$ and $\Delta_{1} f$ denote the Hessian and Laplacian of $f$ on $M_{1}$ respectively, while $H^{h}$ and $\Delta h$ denote the Hessian and Laplacian of $h$ on $\bar{M}$, respectively. In calculations of the next proposition (and its analogues in Subsections 4.1 and 4.2), there is a difference of one minus sign with the results in paper [11]. The reason for this is that we adhere to the Riemann curvature tensor and contruction rules given in Notations 1-(iii).

Lemma 2.2. [11] The non-zero components of the Riemannian curvature of $(\bar{M}, \bar{g})$ are given by:

1. $\bar{R}\left(X_{1}, Y_{1}\right) Z_{1}=R_{1}\left(X_{1}, Y_{1}\right) Z_{1}$,

2. $\bar{R}\left(X_{2}, Y_{2}\right) Z_{2}=R_{2}\left(X_{2}, Y_{2}\right) Z_{2}-\left\|\operatorname{grad}_{1} f\right\|^{2}\left[g_{2}\left(Y_{2}, Z_{2}\right) X_{2}-g_{2}\left(X_{2}, Z_{2}\right) Y_{2}\right]$,

3. $\bar{R}\left(X_{1}, Y_{2}\right) Z_{1}=\frac{1}{f} H_{1}^{f}\left(X_{1}, Z_{1}\right) Y_{2}$,

4. $\bar{R}\left(X_{1}, Y_{2}\right) Z_{2}=-f g_{2}\left(Y_{2}, Z_{2}\right) \nabla_{X_{1}}^{1} \operatorname{grad}_{1} f$,

5. $\bar{R}\left(X_{i}, Y_{3}\right) Z_{j}=\frac{1}{h} H^{h}\left(X_{i}, Z_{j}\right) Y_{3}$, for $i, j=1,2$,

6. $\bar{R}\left(X_{i}, Y_{3}\right) Z_{3}=-h g_{3}\left(Y_{3}, Z_{3}\right) \bar{\nabla}_{X_{i}}$ gradh, $i=1,2$,

7. $\bar{R}\left(X_{3}, Y_{3}\right) Z_{3}=R_{3}\left(X_{3}, Y_{3}\right) Z_{3}-\|$ gradh $\|^{2}\left[g_{3}\left(Y_{3}, Z_{3}\right) X_{3}-g_{3}\left(X_{3}, Z_{3}\right) Y_{3}\right]$.

Lemma 2.3. [11] The non-zero components of the Ricci curvature of $(\bar{M}, \bar{g})$ are given by:

1. $\operatorname{Ric}\left(X_{1}, Y_{1}\right)=\operatorname{Ric}_{1}\left(X_{1}, Y_{1}\right)-\frac{m_{2}}{f} H_{1}^{f}\left(X_{1}, Y_{1}\right)-\frac{m_{3}}{h} H^{\mathrm{h}}\left(X_{1}, Y_{1}\right)$,

2. $\operatorname{Ric}\left(X_{2}, Y_{2}\right)=\operatorname{Ric}_{2}\left(X_{2}, Y_{2}\right)-\mathrm{f}^{\sharp} \mathrm{g}_{2}\left(\mathrm{X}_{2}, \mathrm{Y}_{2}\right)-\frac{\mathrm{m}_{3}}{\mathrm{~h}} \mathrm{H}^{\mathrm{h}}\left(\mathrm{X}_{2}, \mathrm{Y}_{2}\right)$,

3. $\operatorname{Ric}\left(X_{3}, Y_{3}\right)=\operatorname{Ric}_{3}\left(\mathrm{X}_{3}, \mathrm{Y}_{3}\right)-\mathrm{h}^{\sharp} \mathrm{g}_{3}\left(\mathrm{X}_{3}, \mathrm{Y}_{3}\right)$,

where $f^{\sharp}=f \Delta_{1} f+\left(m_{2}-1\right) \|$ grad $_{1} f \|^{2}$ and $h^{\sharp}=h \Delta h+\left(m_{3}-1\right) \|$ gradh $\|^{2}$.

Lemma 2.4. [11] The relation between the scalar curvature $r$ of $(\bar{M}, \bar{g})$ and the scalar curvatures $r_{i}$ of $\left(M_{i}, g_{i}\right)$ is given by:

$$
\bar{r}=r_{1}+\frac{r_{2}}{f^{2}}+\frac{r_{3}}{h^{2}}-\frac{2 m_{2}}{f} \Delta_{1} f-\frac{2 m_{3}}{h} \Delta h-\frac{m_{2}\left(m_{2}-1\right)}{f^{2}}\left\|g r a d_{1} f\right\|^{2}-\frac{m_{3}\left(m_{3}-1\right)}{h^{2}}\|g r a d h\|^{2} .
$$

\subsection{Concircular Curvature Tensor}

A transformation on a Riemannian manifold $M$, which transforms every geodesic circle of $M$ into a geodesic circle, is called a concircular transformation, [25] (for more [24, 6]). A tensor of type $(1,3)$ on an $n$ dimensional Riemannian manifold $M$ denoted by $\mathcal{C}$ which remains invariant under concircular transformations is introduced by Yano and Kon and given by

$$
\mathcal{C}(X, Y) Z=R(X, Y) Z-\frac{r}{n(n-1)}[g(Y, Z) X-g(X, Z) Y], \quad \forall X, Y, Z \in \chi(M) .
$$

Equivalently, it can be expressed as

$$
\mathcal{C}=R-\frac{r}{n(n-1)} G
$$

where $G=\frac{1}{2}(g \wedge g)$ and $\wedge$ denotes the Kulkarni-Nomizu product. A manifold whose concircular curvature vanishes at every point is called concircularly flat. Thus, we have the following direct geometrical consequences: 
- Every concircularly flat manifold is of constant curvature.

- A semi-Riemannian manifold is called concircular symmetric if the covariant derivative of $\mathcal{C}$ vanishes. By (2.3), we have

$$
\nabla \mathcal{C}=\nabla R-\frac{1}{n(n-1)}(\nabla r) G .
$$

Thus, if $M$ is concircular symmetric, then by (2.4) and the contracted second Bianchi identity, the scalar curvature is constant and so $\nabla R=0$, which means that $M$ is locally symmetric. The converse of this result is also true.

- Again by (2.3), the divergence of the concircular curvature tensor is given by

$$
\operatorname{div} \mathcal{C}=\operatorname{div} R-\frac{d r}{n(n-1)} G .
$$

On the othe hand, the divergence of the Riemannian curvature tensor is given by

$$
\operatorname{div} R(X, Y) Z=\left(\nabla_{X} \operatorname{Ric}\right)(Y, Z)-\left(\nabla_{Y} \operatorname{Ric}\right)(X, Z), \quad \forall X, Y, Z \in \chi(M) .
$$

Thus, by (2.5), (2.6) and the contracted second Bianchi identity, the scalar curvature is again constant and so $\operatorname{div} R=0$, which means that the Ricci tensor is of Codazzi type.

The geometric and the relativistic significance of this tensor has been studied by De, Shenawy and Ünal [12] and Ahsan and Siddiqui [1]. In [12], the concircular flatness, the concircular symmetry and the divergence-free concircular curvature tensor on warped product manifolds have been investigated. Also, the effects of these conditions on the base and the fiber of the warped product are the main fields of the study. Our aim in this paper is to extend these results to the sequential warped product manifolds. In this case the calculations will be much more heavy, but give more general results.

\section{Concircular Geometry and Gradient Solitons on Sequential Warped Products}

\subsection{Concircular Flatness and Gradient Solitons}

In this section, by using the Lemmas $2.2,2.3$ and 2.4, we first provide the description of the concircular curvature tensor on the sequential warped product manifold.

Proposition 3.1. Let $\bar{M}=\left(M_{1} \times_{f} M_{2}\right) \times_{h} M_{3}$ be an n-dimensional sequential warped product endowed with the metric $\bar{g}=\left(g_{1} \oplus f^{2} g_{2}\right) \oplus h^{2} g_{3}$ and let $X_{i}, Y_{i}, Z_{i} \in \chi\left(M_{i}\right)$, for $i=1,2,3$. Then, all non-zero components of the concircular curvature tensor $\mathcal{C}$ on $\bar{M}$ are given by:

$$
\begin{aligned}
\mathcal{C}\left(X_{1}, Y_{1}\right) Z_{1} & =R_{1}\left(X_{1}, Y_{1}\right) Z_{1}-\frac{\bar{r}}{n(n-1)}\left[g_{1}\left(Y_{1}, Z_{1}\right) X_{1}-g_{1}\left(X_{1}, Z_{1}\right) Y_{1}\right], \\
\mathcal{C}\left(X_{2}, Y_{2}\right) Z_{2} & =R_{2}\left(X_{2}, Y_{2}\right) Z_{2}-\left(\left\|g r a d_{1} f\right\|^{2}+\frac{\bar{r} f^{2}}{n(n-1)}\right)\left[g_{2}\left(Y_{2}, Z_{2}\right) X_{2}-g_{2}\left(X_{2}, Z_{2}\right) Y_{2}\right], \\
\mathcal{C}\left(X_{1}, Y_{2}\right) Z_{1} & =\frac{1}{f} H_{1}^{f}\left(X_{1}, Z_{1}\right) Y_{2}+\frac{\bar{r}}{n(n-1)} g_{1}\left(X_{1}, Z_{1}\right) Y_{2}, \\
\mathcal{C}\left(X_{1}, Y_{2}\right) Z_{2} & =-f g_{2}\left(Y_{2}, Z_{2}\right) \nabla_{X_{1}}^{1} \text { grad } d_{1} f-\frac{\bar{r} f^{2}}{n(n-1)} g_{2}\left(Y_{2}, Z_{2}\right) X_{1}, \\
\mathcal{C}\left(X_{1}, Y_{3}\right) Z_{1} & =\frac{1}{h} H^{h}\left(X_{1}, Z_{1}\right) Y_{3}+\frac{\bar{r}}{n(n-1)} g_{1}\left(X_{1}, Z_{1}\right) Y_{3}, \\
\mathcal{C}\left(X_{1}, Y_{3}\right) Z_{2} & =\frac{1}{h} H^{h}\left(X_{1}, Z_{2}\right) Y_{3}, \mathcal{C}\left(X_{2}, Y_{3}\right) Z_{1}=\frac{1}{h} H^{h}\left(X_{2}, Z_{1}\right) Y_{3}, \\
\mathcal{C}\left(X_{2}, Y_{3}\right) Z_{2} & =\frac{1}{h} H^{h}\left(X_{2}, Z_{2}\right) Y_{3}+\frac{\bar{r} f^{2}}{n(n-1)} g_{2}\left(X_{2}, Z_{2}\right) Y_{3}, \\
\mathcal{C}\left(X_{1}, Y_{3}\right) Z_{3} & =-h g_{3}\left(Y_{3}, Z_{3}\right) \bar{\nabla}_{X_{i}} \text { gradh- } \frac{\bar{r} h^{2}}{n(n-1)} g_{3}\left(Y_{3}, Z_{3}\right) X_{i}, \quad \text { for } i=1,2, \\
\mathcal{C}\left(X_{3}, Y_{3}\right) Z_{3} & =R_{3}\left(X_{3}, Y_{3}\right) Z_{3}-\left(\|g r a d h\|^{2}+\frac{\bar{r} h^{2}}{n(n-1)}\right)\left[g_{3}\left(Y_{3}, Z_{3}\right) X_{3}-g_{3}\left(X_{3}, Z_{3}\right) Y_{3}\right] .
\end{aligned}
$$


As a consequence of Proposition 3.1, we state:

Theorem 3.1. Let $\bar{M}=\left(M_{1} \times_{f} M_{2}\right) \times_{h} M_{3}$ be an $n$-dimensional $\left(n=m_{1}+m_{2}+m_{3}\right)$ sequential warped product endowed with the metric $\bar{g}=\left(g_{1} \oplus f^{2} g_{2}\right) \oplus h^{2} g_{3}$ and let $X_{i}, Y_{i}, Z_{i} \in \chi\left(M_{i}\right)$, for $i=1,2,3$. Then $(\bar{M}, \bar{g})$ is concircularly flat if and only if the following statements hold:

1. $\left(M_{1}, g_{1}\right)$ is of constant curvature $\kappa_{1}=\frac{\bar{r}}{n(n-1)}$.

2. $\left(M_{2}, g_{2}\right)$ is of constant curvature $\kappa_{2}=\left\|g r a d_{1} f\right\|^{2}+\frac{\bar{r} f^{2}}{n(n-1)}$.

3. For all $X_{1}, Z_{1} \in \chi\left(M_{1}\right), H_{1}^{f}\left(X_{1}, Z_{1}\right)+\frac{\bar{r} f}{n(n-1)} g_{1}\left(X_{1}, Z_{1}\right)=0$.

4. For all $X_{1} \in \chi\left(M_{1}\right)$ and $Z_{2} \in \chi\left(M_{2}\right), H^{h}\left(X_{1}, Z_{2}\right)=0$.

5. For all $X_{2}, Z_{2} \in \chi\left(M_{2}\right), H^{h}\left(X_{2}, Z_{2}\right)+\frac{\bar{r} h f^{2}}{n(n-1)} g_{2}\left(X_{2}, Z_{2}\right)=0$.

6. $\left(M_{3}, g_{3}\right)$ is of constant curvature $\kappa_{3}=\|$ gradh $\|^{2}+\frac{\bar{r} h^{2}}{n(n-1)}$.

A Riemannian manifold $\left(M^{n}, g\right)$ is called a conformal gradient soliton if there exists a non-constant smooth function $f$, called potential of the soliton, such that

$$
\mathrm{H}^{f}=\varphi g,
$$

for some function $\varphi: M^{n} \rightarrow \mathbb{R}$. Tashiro studied in [23] complete Riemannian manifolds admitting a vector field $\nabla f$ satisfying equation (3.2). Then, in [9] Cheeger and Colding gave the solutions of the equation (3.2) and they obtained the characterization of warped product manifolds. Also, the equation (3.2) is the special case of gradient Ricci soliton structure, that has been studied extensively on the warped product manifold in [21]. With the help of this definition and Theorem 3.1-(3), we express the following:

Theorem 3.2. Let $\bar{M}=\left(M_{1} \times_{f} M_{2}\right) \times_{h} M_{3}$ be a sequential warped product endowed with the metric $\bar{g}=\left(g_{1} \oplus f^{2} g_{2}\right) \oplus$ $h^{2} g_{3}$. If $(\bar{M}, \bar{g})$ is concircularly flat, then $\left(M_{1}, g_{1}\right)$ is a conformal gradient soliton whose potential function is the warping function $f$ and $\varphi=-\frac{\bar{r} f}{n(n-1)}$.

From Theorem 3.1-(4) and Lemma 2.1, we have $f^{2} X_{1}(\ln f) g_{2}\left(\operatorname{grad} h_{2}, Z_{2}\right)=0$, for all $Z_{2} \in \chi\left(M_{2}\right)$, where $\operatorname{grad} h=\operatorname{grad} h_{1}+\operatorname{grad} h_{2}$ and $\operatorname{grad} h_{i}$ denotes the tangential and the normal parts of $h$ on $M_{i}(i=1,2)$, respectively. Since $f$ is non constant, positive function, the last equation yields that $\operatorname{grad} h_{2}=0$. Therefore, we can state:

Corollary 3.1. Let $\bar{M}=\left(M_{1} \times{ }_{f} M_{2}\right) \times_{h} M_{3}$ be a sequential warped product endowed with the metric $\bar{g}=\left(g_{1} \oplus f^{2} g_{2}\right) \oplus$ $h^{2} g_{3}$. If $(\bar{M}, \bar{g})$ is concircularly flat, then the warping function $h$ depends only on $M_{1}$.

From Theorem 3.1's (3)-(5), we have

$$
\Delta_{1} f=-m_{1} \kappa_{1} f \text { and } \Delta h=-\left(m_{1}+m_{2} f^{2}\right) \kappa_{1} h .
$$

If we additionally assume that $M_{1}$ and $M_{1} \times_{f} M_{2}$ are compact and $\bar{r} \leq 0$ (or $\bar{r} \geq 0$ ), then by (3.3) we get $\Delta_{1} f$ and $\Delta h$ do not change the signs. Then it follows from Hopf's Lemma that the warping functions $f$ and $h$ become constants. Therefore we can state the following rigidity result:

Corollary 3.2. Let $\bar{M}=\left(M_{1} \times_{f} M_{2}\right) \times_{h} M_{3}$ be a sequential warped product endowed with the metric $\bar{g}=\left(g_{1} \oplus f^{2} g_{2}\right) \oplus$ $h^{2} g_{3}$, where $M_{1}$ and $M_{2}$ are compact. If $(\bar{M}, \bar{g})$ is concircularly flat such that $\bar{r} \leq 0$ (or $\bar{r} \geq 0$ ), then it reduces to a simply direct product.

A smooth manifold $\left(M^{n}, g\right)(n>2)$ is said to be a generalized quasi Einstein manifold in the sense of Catino [7] if there exist three smooth functions $\varphi, \alpha$ and $\lambda$ such that

$$
\operatorname{Ric}+\mathrm{H}^{\varphi}-\alpha d \varphi \otimes d \varphi=\lambda g
$$

and it is denoted by $\left(M^{n}, g, \varphi, \alpha, \lambda\right)$. There are some different subclasses of this equation that define many important manifolds:

(i) If $\alpha=\frac{1}{m}$, for positive integer $0<m<\infty$ then $M$ is called an $m$-generalized quasi Einstein manifold. 
(ii) If $\alpha=0$, then $M$ is called a gradient almost Ricci soliton, [3] and it is denoted by $(M, g, \varphi, \lambda)$ and $\varphi$ is called the potential function.

(iii) Another particular case of (3.4) can be written as

$$
\operatorname{Ric}+\psi \mathrm{H}^{\varphi}=\lambda g .
$$

This structure is said to be a $\psi$-almost gradient Ricci soliton [26] and briefly denoted by $(M, g, \varphi, \psi, \lambda)$. Note that, for every smooth function $\varphi$, the following relation can be verified:

$$
H^{\ln \varphi}=\frac{1}{\varphi} H^{\varphi}-\frac{1}{\varphi^{2}} \mathrm{~d} \varphi \otimes \mathrm{d} \varphi
$$

By defining a function $\phi=e^{\frac{-\varphi}{m}}$, we get $\frac{m}{\phi} \mathrm{H}^{\phi}=-\mathrm{H}^{\varphi}+\frac{1}{m} d \varphi \otimes d \varphi$. Thus using (3.6), (3.4) can be written as

$$
\text { Ric }-\frac{m}{\phi} \mathrm{H}^{\phi}=\lambda g .
$$

Hence, any $m$-generalized quasi Einstein manifold is a $\left(\frac{-m}{\phi}\right)$-almost gradient Ricci soliton. In [15], the author gave some classifications for half-conformally flat $f$-almost gradient Ricci solitons and physical applications of them in standard static spacetimes.

Now, we prove the following result:

Theorem 3.3. Let $\bar{M}=\left(M_{1} \times_{f} M_{2}\right) \times_{h} M_{3}$ be a sequential warped product endowed with the metric $\bar{g}=\left(g_{1} \oplus f^{2} g_{2}\right) \oplus$ $h^{2} g_{3}$. If $(\bar{M}, \bar{g}, \varphi, \lambda)$ is a gradient almost Ricci soliton, then

1. the potential function $\varphi$ depends only on $\left(M_{1}, g_{1}\right)$ and it satisfies

$$
\operatorname{Ric}_{1}-\frac{\mathrm{m}_{2}}{\mathrm{f}} \mathrm{H}_{1}^{\mathrm{f}}-\frac{\mathrm{m}_{3}}{\mathrm{~h}} \mathrm{H}^{\mathrm{h}}+\mathrm{H}_{1}^{\varphi}=\lambda \mathrm{g}_{1} .
$$

2. $\left(M_{2}, g_{2}, \tilde{\varphi}, \tilde{\alpha}, \tilde{\lambda}\right)$ is a generalized quasi Einstein manifold, where $\tilde{\varphi}=-m_{3} \ln h, \tilde{\alpha}=-1 / m_{3}$ and $\tilde{\lambda}=\lambda f^{2}+f^{\sharp}-$ $f \nabla \varphi(f)$.

3. $\left(M_{3}, g_{3}, \varphi, \lambda h^{2}+h^{\sharp}-h \nabla \varphi(h)\right)$ is a gradient almost Ricci soliton.

Proof. Let $(\bar{M}, \bar{g}, \varphi, \lambda)$ be a gradient almost Ricci soliton. Then we have

$$
\operatorname{Ric}+H^{\varphi}=\lambda \bar{g} .
$$

For a smooth function $\varphi$ on a sequential warped product $\bar{M}=\left(M_{1} \times_{f} M_{2}\right) \times_{h} M_{3}$, we define, $H_{i}^{\varphi}\left(X_{i}, Y_{i}\right)=$ $X_{i} Y_{i}(\varphi)-\left(\nabla_{X_{i}}^{i} Y_{i}\right)(\varphi)$ for all $X_{i}, Y_{i} \in \mathfrak{L}\left(M_{i}\right)$, for $i=1,2,3$. Then by using Lemma 2.1, the Hessian tensor $H^{\varphi}$ of $\varphi$ on a sequential warped product $\bar{M}=\left(M_{1} \times_{f} M_{2}\right) \times_{h} M_{3}$ satisfies

$$
\begin{aligned}
& \text { (1) } H^{\varphi}\left(X_{1}, Y_{1}\right)=H_{1}^{\varphi}\left(X_{1}, Y_{1}\right), \\
& \text { (2) } H^{\varphi}\left(X_{1}, Y_{2}\right)=X_{1}(\ln f) Y_{2}(\varphi), \\
& \text { (3) } H^{\varphi}\left(X_{1}, Y_{3}\right)=X_{1}(\ln h) Y_{3}(\varphi), \\
& \text { (4) } H^{\varphi}\left(X_{2}, Y_{2}\right)=f \nabla \varphi(f) g_{2}\left(X_{2}, Y_{2}\right)+H_{2}^{\varphi}\left(X_{2}, Y_{2}\right), \\
& \text { (5) } H^{\varphi}\left(X_{2}, Y_{3}\right)=X_{2}(\ln h) Y_{3}(\varphi), \\
& \text { (6) } H^{\varphi}\left(X_{3}, Y_{3}\right)=h \nabla \varphi(h) g_{3}\left(X_{3}, Y_{3}\right)+H_{3}^{\varphi}\left(X_{3}, Y_{3}\right) .
\end{aligned}
$$

By using the fact that the warping functions $f$ and $h$ are defined on $M_{1}$ and $M_{1} \times_{f} M_{2}$, respectively, and (2),(3) and (5) of (3.10) into (3.9) we obtain $Y_{i}(\varphi)=0$, for all $Y_{i} \in \chi\left(M_{i}\right), i=2,3$. This gives us the potential function $\varphi$ is defined only on $M_{1}$. Also combining Lemma 2.3-(1) and (3.10)-(1) with (3.9), we get (3.8). Then, by using Lemma 2.3-(2) and (3.10)-(4) into (3.9), we get

$$
\operatorname{Ric}_{2}-\frac{\mathrm{m}_{3}}{\mathrm{~h}} \mathrm{H}^{\mathrm{h}}=\left(\mathrm{f}^{\sharp}+\lambda \mathrm{f}^{2}-\mathrm{f} \nabla \varphi(\mathrm{f})\right) \mathrm{g}_{2} .
$$

By virtue of (3.6), (3.11) proves the second assertion. Finally, using Lemma 2.3-(3) and (3.10)-(6) into (3.9), we get the third assertion. 


\subsection{Concircular Symmetry}

Now, we investigate some symmetries on sequential warped products. By (2.4), it is known that if the manifold is concircular symmetric, then it is also locally symmetric with constant scalar curvature. Thus, by using Lemma 2.2, we prove the following:

Theorem 3.4. Let $\bar{M}=\left(M_{1} \times_{f} M_{2}\right) \times_{h} M_{3}$ be a sequential warped product endowed with the metric $\bar{g}=\left(g_{1} \oplus f^{2} g_{2}\right) \oplus$ $h^{2} g_{3}$. If $(\bar{M}, \bar{g})$ is concircular symmetric, then the followings hold:

1. $M_{1}, M_{2}$ and $M_{3}$ are locally symmetric manifolds.

2. $\mathcal{F}=\frac{1}{f} H_{1}^{f}$ and $\mathcal{H}=\frac{1}{h} H^{f}$ are parallel.

3. $R_{1}\left(\operatorname{grad}_{1} f, X_{1}\right) Z_{1}=\mathcal{F}\left(X_{1}, Z_{1}\right) \operatorname{grad}_{1} f+Z_{1}(\ln f) \nabla_{X_{1}}^{1} \operatorname{grad}^{1} f$.

4. $W_{1}(\ln f) R_{2}\left(X_{2}, Y_{2}\right) Z_{2}-W_{1}\left(\left\|\operatorname{grad}_{1} f\right\|^{2}\right) G_{2}\left(X_{2}, Y_{2}\right) Z_{2}=-f g_{2}\left(Y_{2}, Z_{2}\right) \bar{\nabla}_{W_{1}} \operatorname{grad}_{1} f$.

Proof. Since $(\bar{M}, \bar{g})$ is concircular symmetric, for all $X_{i}, Y_{i}, Z_{i}, W_{i} \in \chi\left(M_{i}\right)(i=1,2,3),\left(\bar{\nabla}_{W_{i}} \mathcal{C}\right)\left(X_{i}, Y_{i}\right) Z_{i}=0$. By using all components of the Riemannian curvature tensor given in Lemma 2.2, we get:

$$
\left(\bar{\nabla}_{W_{i}} R_{i}\right)\left(X_{i}, Y_{i}\right) Z_{i}=0, \text { for all } X_{i}, Y_{i}, Z_{i}, W_{i} \in \chi\left(M_{i}\right),
$$

which proves (1). Also, by $\left(\bar{\nabla}_{W_{1}} R_{i}\right)\left(X_{1}, Y_{2}\right) Z_{1}=0$ and $\left(\bar{\nabla}_{W_{1}} R_{i}\right)\left(X_{i}, Y_{3}\right) Z_{i}=0,(i=1,2)$, we get

$$
\left(\bar{\nabla}_{W_{1}} \frac{1}{f} H_{1}^{f}\right)\left(X_{1}, Y_{1}\right)=0, \text { for all } X_{1}, Y_{1} \in \chi\left(M_{1}\right)
$$

and

$$
\left(\bar{\nabla}_{W_{i}} \frac{1}{h} H^{h}\right)\left(X_{i}, Y_{i}\right)=0, \text { for all } X_{i}, Y_{i} \in \chi\left(M_{i}\right), i=1,2 .
$$

respectively. Thus the last two equations prove the assertion (2). Using the definitions of $\mathcal{F}$ and $\mathcal{H}$ and Lemma 2.1's third item into $\left(\bar{\nabla}_{W_{2}} \bar{R}\right)\left(X_{1}, Y_{2}\right) Z_{1}=0$ and $\left(\bar{\nabla}_{W_{1}} \bar{R}\right)\left(X_{2}, Y_{2}\right) Z_{2}=0$, we get (3) and (4), respectively. Hence the proof is completed.

\subsection{Divergence-Free Concircular Curvature Tensor}

First let $\mathcal{C}_{i}$ be the concircular curvature tensor on $M_{i}$, for $i=1,2$. If the concircular curvature tensor $\mathcal{C}$ of a sequential warped product $\bar{M}=\left(M_{1} \times_{f} M_{2}\right) \times_{h} M_{3}$ is divergence-free, then $\operatorname{div} \bar{R}=0$ so the Ricci tensor is of Codazzi type. Thus, the tensor $T$ defined by

$$
T(X, Y, Z)=\left(\nabla_{X} \operatorname{Ric}\right)(Y, Z)-\left(\nabla_{Y} \operatorname{Ric}\right)(X, Z), \quad \forall X, Y, Z \in \chi(\bar{M})
$$

vanishes identically.

Now, under this construction, we need to analyse the following cases:

(i) Let $X_{1}, Y_{1}, Z_{1} \in \chi\left(M_{1}\right)$. As $T\left(X_{1}, Y_{1}, Z_{1}\right)=0$, it follows from Lemma 2.3 that $\operatorname{div} \mathcal{C}_{1}=0$ if and only if the following equation holds:

$$
\begin{aligned}
& \frac{m_{2}}{f}\left[R_{1}\left(X_{1}, Y_{1}, Z_{1}, \operatorname{grad}_{1} f\right)+Y_{1}(f) \mathcal{F}\left(X_{1}, Z_{1}\right)-X_{1}(f) \mathcal{F}\left(Y_{1}, Z_{1}\right)\right] \\
& =\frac{m_{3}}{h}\left[R_{1}\left(X_{1}, Y_{1}, \operatorname{gradh}, Z_{1}\right)-Y_{1}(h) \mathcal{H}\left(X_{1}, Z_{1}\right)+X_{1}(h) \mathcal{H}\left(Y_{1}, Z_{1}\right)\right] .
\end{aligned}
$$

(ii) Let $X_{2}, Y_{2}, Z_{2} \in \chi\left(M_{2}\right)$. As $T\left(X_{2}, Y_{2}, Z_{2}\right)=0$, it follows from Lemma 2.3 and the Ricci identity that $\operatorname{div} \mathcal{C}_{2}=0$ if and only if the following equation holds:

$$
R_{2}\left(X_{2}, Y_{2}, \text { gradh }, Z_{2}\right)=Y_{2}(\ln h) \mathcal{H}\left(X_{2}, Z_{2}\right)-X_{2}(\ln h) \mathcal{H}\left(Y_{2}, Z_{2}\right) .
$$

(iii) Let $X_{3}, Y_{3}, Z_{3} \in \chi\left(M_{3}\right)$. As $T\left(X_{3}, Y_{3}, Z_{3}\right)=0$ and $h \in C^{\infty}\left(M_{1} \times M_{2}\right)$, by Lemma 2.3, we always have $\operatorname{div} \mathcal{C}_{3}=0$. 
(iv) As $T\left(X_{1}, Y_{2}, Z_{2}\right)=0$, by virtue of Lemma 2.3, (as $f$ is non-constant, $\left.X_{1}(\ln f) \neq 0\right)$ we have

$$
\begin{aligned}
\operatorname{Ric}_{2}\left(\mathrm{Y}_{2}, \mathrm{Z}_{2}\right) & -\frac{m_{3}}{h}\left(1+\frac{X_{1}(\ln h)}{2 X_{1}(\ln f)}\right) \mathrm{H}^{h}\left(Y_{2}, Z_{2}\right)-\frac{m_{3} f^{2}}{h} H_{2}^{h}\left(Y_{2}, Z_{2}\right) \\
= & -\left[\frac{X_{1}\left(f^{\sharp}\right)}{2 X_{1}(\ln f)}-\frac{m_{3}}{2 h X_{1}(\ln f)} X_{1}(\operatorname{gradh}(f))\right] g_{2}\left(Y_{2}, Z_{2}\right) .
\end{aligned}
$$

(v) As $T\left(X_{1}, Y_{3}, Z_{3}\right)=0$, by virtue of Lemma 2.3 , (as $h$ is non-constant) we have

$$
\operatorname{Ric}_{3}\left(Y_{3}, Z_{3}\right)=-\frac{X_{1}\left(h^{\sharp}\right)}{2 X_{1}(\ln h)} g_{3}\left(Y_{3}, Z_{3}\right)
$$

provided that $X_{1}(h) \neq 0$.

(vi) As $T\left(X_{2}, Y_{1}, Z_{1}\right)=0$, by virtue of Lemma 2.3 and $X_{1}(h) \neq 0$, we obtain $X_{2}(h)=0$, for all $X_{2} \in \chi\left(M_{2}\right)$. That is, $h$ depends only on $M_{1}$. Combining this result with the equation (3.18), we get

$$
\operatorname{Ric}_{2}\left(\mathrm{Y}_{2}, \mathrm{Z}_{2}\right)+\psi \mathrm{H}^{\mathrm{h}}\left(\mathrm{Y}_{2}, \mathrm{Z}_{2}\right)=\lambda \mathrm{g}_{2}\left(\mathrm{Y}_{2}, \mathrm{Z}_{2}\right)
$$

which is the fundamental equation of the $\psi$-almost gradient Ricci soliton defined in (3.5), where

$$
\psi=-\frac{m_{3}}{h}\left(1+\frac{X_{1}(\ln h)}{2 X_{1}(\ln f)}\right), \quad \text { and } \lambda=-\frac{X_{1}\left(f^{\sharp}\right)}{2 X_{1}(\ln f)}-\frac{m_{3}}{2 h X_{1}(\ln f)} X_{1}(\operatorname{gradh}(f)) .
$$

Moreover, all the other rest cases of the components of $T$ vanish directly. Hence we get the following:

Theorem 3.5. Let $\bar{M}=\left(M_{1} \times{ }_{f} M_{2}\right) \times_{h} M_{3}$ be a sequential warped product endowed with the metric $\bar{g}=\left(g_{1} \oplus f^{2} g_{2}\right) \oplus$ $h^{2} g_{3}$. If $(\bar{M}, \bar{g})$ has divergence-free concircular curvature tensor, then:

1. $M_{1}$ has divergence-free concircular curvature tensor if and only if (3.16) holds.

2. $M_{2}$ has divergence-free concircular curvature tensor if and only if (3.17) holds.

3. $M_{3}$ is Einstein manifold and so it always has divergence-free concircular curvature tensor.

4. The warping function $h$ depends only on $M_{1}$.

5. $\left(M_{2}, g_{2}, h, \psi, \lambda\right)$ is a $\psi$-almost gradient Ricci soliton, where $\psi$ and $\lambda$ are given by (3.21).

\section{Some Applications of Sequential Warped Product Spacetimes}

Generalized Robertson-Walker spacetime is a warped product spacetime whose base is an open interval $I$ of $\mathbb{R}$ with its usual metric reversed $\left(I,-\mathrm{d} t^{2}\right)$, the fiber is an $m$-dimensional connected Riemannian manifold $(M, g)$ and the warping function is any positive smooth fuction $f>0$ on $I$, [19]. More precisely, the generalized Robertson-Walker spacetime is the product manifold $\bar{M}=I \times{ }_{f} M$ endowed with the Lorentzian metric $\bar{g}=$ $-\mathrm{d} t^{2}+f^{2}(t) g, f \in \mathcal{C}_{>0}^{\infty}(I)$. They have been studied by many authors, such as Mantica, Molinari and De [17], Mantica , Suh, and De [18], Chen [10] and others.

Standard static spacetime which can be actually considered as a Lorentzian warped product where the warping function defined on the fiber which is a Riemannian manifold and acts on the negative definite metric on the base which is an open interval of real numbers. These type of metrics also play an important role to find the solutions of the Einstein field equations so they have been previously studied by many authors, such as Dobarro and Ünal [13] and Allison [2]. Moreover, first in [11], these two structures have been studied on the sequential warped product structure and then in [11] and [16] the necessary and sufficient conditions are given for the existence of Einstein and quasi Einstein manifolds on the sequential warped products, respectively.

\subsection{Sequential Generalized Robertson-Walker Spacetimes}

Let $\bar{M}=\left(I \times_{f} M_{2}\right) \times_{h} M_{3}$ be a sequential generalized Robertson-Walker warped product spacetime endowed with the metric $\bar{g}=\left(-\mathrm{d} t^{2} \oplus f^{2} g_{2}\right) \oplus h^{2} g_{3}$ and let $X_{i}, Y_{i}, Z_{i} \in \chi\left(M_{i}\right)$, for $i=2,3$. Then, by direct applications of Lemmas presented in Subsection 2.1, we obtain the followings: 
Lemma 4.1. [11] The non-zero components of the Levi-Civita connection on $(\bar{M}, \bar{g})$ are given by:

1. $\bar{\nabla}_{\partial t} X_{i}=\bar{\nabla}_{X_{i}} \partial t=\frac{\dot{f}}{f} X_{i}, i=2,3$,

2. $\bar{\nabla}_{X_{2}} Y_{2}=\nabla_{X_{2}}^{2} Y_{2}-f \dot{f} g_{2}\left(X_{2}, Y_{2}\right) \partial t$,

3. $\bar{\nabla}_{X_{2}} X_{3}=\bar{\nabla}_{X_{3}} X_{2}=X_{2}(\ln h) X_{3}$

4. $\bar{\nabla}_{X_{3}} Y_{3}=\nabla_{X_{3}}^{3} Y_{3}-h g_{3}\left(X_{3}, Y_{3}\right)$ gradh.

Lemma 4.2. [11] The non-zero components of the Riemannian curvature of $(\bar{M}, \bar{g})$ are given by:

1. $\bar{R}\left(X_{2}, Y_{2}\right) Z_{2}=R_{2}\left(X_{2}, Y_{2}\right) Z_{2}+(\dot{f})^{2}\left[g_{2}\left(Y_{2}, Z_{2}\right) X_{2}-g_{2}\left(X_{2}, Z_{2}\right) Y_{2}\right]$,

2. $\bar{R}\left(\partial_{t}, Y_{2}\right) \partial_{t}=-\frac{\ddot{f}}{f} Y_{2}$

3. $\bar{R}\left(\partial_{t}, Y_{3}\right) \partial_{t}=-\frac{1}{h} \frac{\partial^{2} h}{\partial t^{2}} Y_{3}$

4. $\bar{R}\left(\partial_{t}, Y_{2}\right) Z_{2}=-f \ddot{f} g_{2}\left(Y_{2}, Z_{2}\right) \partial_{t}$,

5. $\bar{R}\left(X_{2}, Y_{3}\right) Z_{2}=\frac{1}{h} H^{h}\left(X_{2}, Z_{2}\right) Y_{3}$

6. $\bar{R}\left(\partial_{t}, Y_{3}\right) Z_{3}=-h g_{3}\left(Y_{3}, Z_{3}\right) \bar{\nabla}_{\partial_{t}}$ gradh,

7. $\bar{R}\left(X_{2}, Y_{3}\right) Z_{3}=-h g_{3}\left(Y_{3}, Z_{3}\right) \bar{\nabla}_{X_{2}}$ gradh,

8. $\bar{R}\left(X_{3}, Y_{3}\right) Z_{3}=R_{3}\left(X_{3}, Y_{3}\right) Z_{3}-\|\operatorname{gradh}\|^{2}\left[g_{3}\left(Y_{3}, Z_{3}\right) X_{3}-g_{3}\left(X_{3}, Z_{3}\right) Y_{3}\right]$.

Lemma 4.3. [11] The non-zero components of the Ricci curvature of $(\bar{M}, \bar{g})$ are given by:

1. $\overline{\operatorname{Ric}}\left(\partial_{t}, \partial_{t}\right)=\frac{m_{2}}{f} \ddot{f}+\frac{m_{3}}{h} \frac{\partial^{2} h}{\partial t^{2}}$,

2. $\overline{\operatorname{Ric}}\left(X_{2}, Y_{2}\right)=\operatorname{Ric}_{2}\left(X_{2}, Y_{2}\right)-f^{\sharp} g_{2}\left(X_{2}, Y_{2}\right)-\frac{m_{3}}{h} H^{h}\left(X_{2}, Y_{2}\right)$,

3. $\overline{\operatorname{Ric}}\left(X_{3}, Y_{3}\right)=\operatorname{Ric}_{3}\left(\mathrm{X}_{3}, \mathrm{Y}_{3}\right)-\mathrm{h}^{\sharp} \mathrm{g}_{3}\left(\mathrm{X}_{3}, \mathrm{Y}_{3}\right)$,

where $f^{\sharp}=-f \ddot{f}-\left(m_{2}-1\right)(\dot{f})^{2}$ and $h^{\sharp}=h \Delta h+\left(m_{3}-1\right) \|$ gradh $\|^{2}$.

Lemma 4.4. [11] The relation between the scalar curvature $r$ of $(\bar{M}, \bar{g})$ and the scalar curvatures $r_{i}$ of $\left(M_{i}, g_{i}\right)$ is given by:

$$
\bar{r}=\frac{r_{2}}{f^{2}}+\frac{r_{3}}{h^{2}}+\frac{2 m_{2}}{f} \ddot{f}-\frac{2 m_{3}}{h} \Delta h-\frac{m_{2}\left(m_{2}-1\right)}{f^{2}}(\dot{f})^{2}-\frac{m_{3}\left(m_{3}-1\right)}{h^{2}} \| \text { gradh } \|^{2} .
$$

By virtue of the Lemmas 4.2,4.3 and 4.4, now we provide the description of the concircular curvature tensor on the sequential generalized Robertson-Walker spacetime:

Proposition 4.1. Let $\bar{M}=\left(I \times{ }_{f} M_{2}\right) \times_{h} M_{3}$ be an $n\left(=m_{2}+m_{3}+1\right)$-dimensional sequential generalized RobertsonWalker spacetime endowed with the metric $\bar{g}=\left(-d t^{2} \oplus f^{2} g_{2}\right) \oplus h^{2} g_{3}$ and let $X_{i}, Y_{i}, Z_{i} \in \chi\left(M_{i}\right)$, for $i=2,3$. Then, all 
non-zero components of the concircular curvature tensor $\mathcal{C}$ on $\bar{M}$ are given by:

$$
\begin{aligned}
\mathcal{C}\left(X_{2}, Y_{2}\right) Z_{2} & =R_{2}\left(X_{2}, Y_{2}\right) Z_{2}+\left((\dot{f})^{2}-\frac{\bar{r} f^{2}}{n(n-1)}\right)\left[g_{2}\left(Y_{2}, Z_{2}\right) X_{2}-g_{2}\left(X_{2}, Z_{2}\right) Y_{2}\right] \\
\mathcal{C}\left(\partial_{t}, Y_{2}\right) \partial_{t} & =-\left(\frac{\ddot{f}}{f}+\frac{\bar{r}}{n(n-1)}\right) Y_{2}, \\
\mathcal{C}\left(\partial_{t}, Y_{2}\right) Z_{2} & =-\left(f \ddot{f}+\frac{\bar{r} f^{2}}{n(n-1)}\right) g_{2}\left(Y_{2}, Z_{2}\right) \partial_{t}, \\
\mathcal{C}\left(\partial_{t}, Y_{3}\right) \partial_{t} & =-\left(\frac{1}{h} \frac{\partial^{2} h}{\partial t^{2}}+\frac{\bar{r}}{n(n-1)}\right) Y_{3}, \quad \mathcal{C}\left(\partial_{t}, Y_{3}\right) Z_{2}=f \dot{f} Z_{2}(\ln h), \\
\mathcal{C}\left(X_{2}, Y_{3}\right) Z_{2} & =\frac{1}{h} H^{h}\left(X_{2}, Z_{2}\right) Y_{3}+\frac{\bar{r} f^{2}}{n(n-1)} g_{2}\left(X_{2}, Z_{2}\right) Y_{3}, \\
\mathcal{C}\left(\partial_{t}, Y_{3}\right) Z_{3} & =-h \ddot{f} g_{3}\left(Y_{3}, Z_{3}\right) \partial_{t}-\frac{\bar{r} h^{2}}{n(n-1)} g_{3}\left(Y_{3}, Z_{3}\right) \partial_{t}, \\
\mathcal{C}\left(X_{2}, Y_{3}\right) Z_{3} & =-h g_{3}\left(Y_{3}, Z_{3}\right) \bar{\nabla} X_{2} g r a d h-\frac{\bar{r} h^{2}}{n(n-1)} g_{3}\left(Y_{3}, Z_{3}\right) X_{2}, \\
\mathcal{C}\left(X_{3}, Y_{3}\right) Z_{3} & =R_{3}\left(X_{3}, Y_{3}\right) Z_{3}-\left(\|g r a d h\|^{2}+\frac{\bar{r} h^{2}}{n(n-1)}\right)\left[g_{3}\left(Y_{3}, Z_{3}\right) X_{3}-g_{3}\left(X_{3}, Z_{3}\right) Y_{3}\right]
\end{aligned}
$$

Considering the vanishing components of the concircular curvature tensor, the result of Proposition 4.1 is expressed as follows:

Theorem 4.1. Let $\bar{M}=\left(I \times_{f} M_{2}\right) \times_{h} M_{3}$ be an $n\left(=m_{2}+m_{3}+1\right)$-dimensional sequential generalized RobertsonWalker spacetime endowed with the metric $\bar{g}=\left(-d t^{2} \oplus f^{2} g_{2}\right) \oplus h^{2} g_{3}$ and let $X_{i}, Y_{i}, Z_{i} \in \chi\left(M_{i}\right)$, for $i=2,3$. Then, $(\bar{M}, \bar{g})$ is concircularly flat if and only if the following statements hold:

1. $\left(M_{2}, g_{2}\right)$ is of constant curvature $\kappa_{2}=\frac{\bar{r} f^{2}}{n(n-1)}-(\dot{f})^{2}$.

2. $\left(M_{3}, g_{3}\right)$ is of constant curvature $\kappa_{3}=\|\operatorname{grad} h\|^{2}+\frac{\bar{r} h^{2}}{n(n-1)}$.

3. $h=f$ and so the warping function $h$ depends only on $M_{1}$.

4. $\ddot{f}+\frac{\bar{r} f}{n(n-1)}=0$ and $\dot{f}+\frac{\bar{r} f}{n(n-1)}=0$ holds.

Theorem 4.2. Let $\bar{M}=\left(I \times_{f} M_{2}\right) \times_{h} M_{3}$ be an $n\left(=m_{2}+m_{3}+1\right)$-dimensional sequential generalized RobertsonWalker spacetime endowed with the metric $\bar{g}=\left(-d t^{2} \oplus f^{2} g_{2}\right) \oplus h^{2} g_{3}$. If $(\bar{M}, \bar{g})$ is concircularly flat, then the warping functions are equal and defined by

$$
f(t)=h(t)=c_{1} e^{t}+c_{2}, \quad c_{1}, c_{2} \in \mathbb{R} .
$$

Proof. From the forth assertion of Theorem 4.1, the warping $f$ satisfies the second order linear differential equation $\ddot{f}-\dot{f}=0$, which has the solution (4.3). Also, from the third assertion of Theorem $4.1, h$ has the same form with $f$.

\subsection{Sequential Standard Static Spacetimes}

Let $\bar{M}=\left(M_{1} \times_{f} M_{2}\right) \times_{h} I$ be a sequential standard static spacetime endowed with the metric $\bar{g}=\left(g_{1} \oplus\right.$ $\left.f^{2} g_{2}\right) \oplus h^{2}\left(-\mathrm{d} t^{2}\right)$ and let $X_{i}, Y_{i}, Z_{i} \in \chi\left(M_{i}\right)$, for $i=1,2$. Then, by direct applications of Lemmas presented in Subsection 2.1, we obtain the followings:

Lemma 4.5. [11] The non-zero components of the Levi-Civita connection on $(\bar{M}, \bar{g})$ are given by:

1. $\bar{\nabla}_{X_{1}} Y_{1}=\nabla_{X_{1}}^{1} Y_{1}$

2. $\bar{\nabla}_{X_{1}} Y_{2}=\bar{\nabla}_{Y_{2}} X_{1}=X_{1}(\ln f) Y_{2}$,

3. $\bar{\nabla}_{X_{2}} Y_{2}=\nabla_{X_{2}}^{2} Y_{2}-f g_{2}\left(X_{2}, Y_{2}\right) \operatorname{grad}_{1} f$,

4. $\bar{\nabla}_{\partial t} X_{i}=\bar{\nabla}_{X_{i}} \partial t=X_{i}(\ln h) \partial_{t}, i=1,2$, 
5. $\bar{\nabla}_{\partial_{t}} \partial_{t}=$ hgradh .

Lemma 4.6. [11] The non-zero components of the Riemannian curvature of $(\bar{M}, \bar{g})$ are given by:

1. $\bar{R}\left(X_{1}, Y_{1}\right) Z_{1}=R_{1}\left(X_{1}, Y_{1}\right) Z_{1}$,

2. $\bar{R}\left(X_{2}, Y_{2}\right) Z_{2}=R_{2}\left(X_{2}, Y_{2}\right) Z_{2}-\left\|\operatorname{grad}_{1} f\right\|^{2}\left[g_{2}\left(Y_{2}, Z_{2}\right) X_{2}-g_{2}\left(X_{2}, Z_{2}\right) Y_{2}\right]$,

3. $\bar{R}\left(X_{1}, Y_{2}\right) Z_{1}=\frac{1}{f} H_{1}^{f}\left(X_{1}, Z_{1}\right) Y_{2}$,

4. $\bar{R}\left(X_{1}, Y_{2}\right) Z_{2}=-f g_{2}\left(Y_{2}, Z_{2}\right) \nabla_{X_{1}}^{1} \operatorname{grad}_{1} f$,

5. $\bar{R}\left(X_{i}, \partial_{t}\right) Z_{j}=\frac{1}{h} H^{h}\left(X_{i}, Z_{j}\right) \partial_{t}, \quad(i, j=1,2)$,

6. $\bar{R}\left(X_{i}, \partial_{t}\right) \partial_{t}=h \bar{\nabla}_{X_{i}}$ gradh, $(i=1,2)$.

Lemma 4.7. [11] The non-zero components of the Ricci curvature of $(\bar{M}, \bar{g})$ are given by:

1. $\overline{\operatorname{Ric}}\left(X_{1}, Y_{1}\right)=\operatorname{Ric}_{1}\left(\mathrm{X}_{1}, \mathrm{Y}_{1}\right)-\frac{\mathrm{m}_{2}}{\mathrm{f}} \mathrm{H}_{1}^{\mathrm{f}}\left(\mathrm{X}_{1}, \mathrm{Y}_{1}\right)-\frac{1}{\mathrm{~h}} \mathrm{H}^{\mathrm{h}}\left(\mathrm{X}_{1}, \mathrm{Y}_{1}\right)$,

2. $\operatorname{Ric}\left(X_{2}, Y_{2}\right)=\operatorname{Ric}_{2}\left(\mathrm{X}_{2}, \mathrm{Y}_{2}\right)-\mathrm{f}^{\sharp} \mathrm{g}_{2}\left(\mathrm{X}_{2}, \mathrm{Y}_{2}\right)-\frac{1}{\mathrm{~h}} \mathrm{H}^{\mathrm{h}}\left(\mathrm{X}_{2}, \mathrm{Y}_{2}\right)$,

3. $\overline{\operatorname{Ric}}\left(\partial_{t}, \partial_{t}\right)=h^{\sharp}$,

where $f^{\sharp}=f \Delta_{1} f+\left(m_{2}-1\right) \|$ grad $_{1} f \|^{2}$ and $h^{\sharp}=h \Delta h$.

Analogous with Subsection 4.1, by virtue of the Lemmas 4.6 and 4.7 , finally we provide the description of the concircular curvature tensor on the sequential standard static spacetime:

Proposition 4.2. Let $\bar{M}=\left(M_{1} \times_{f} M_{2}\right) \times_{h} I$ be an $n\left(=m_{1}+m_{2}+1\right)$-dimensional sequential standard static spacetime endowed with the metric $\bar{g}=\left(g_{1} \oplus f^{2} g_{2}\right) \oplus h^{2}\left(-d t^{2}\right)$ and let $X_{i}, Y_{i}, Z_{i} \in \chi\left(M_{i}\right)$, for $i=1,2$. Then, all nonzero components of the concircular curvature tensor $\mathcal{C}$ on $\bar{M}$ are given by:

$$
\begin{aligned}
\mathcal{C}\left(X_{1}, Y_{1}\right) Z_{1} & =R_{1}\left(X_{1}, Y_{1}\right) Z_{1}-\frac{\bar{r}}{n(n-1)}\left[g_{1}\left(Y_{1}, Z_{1}\right) X_{1}-g_{1}\left(X_{1}, Z_{1}\right) Y_{1}\right], \\
\mathcal{C}\left(X_{2}, Y_{2}\right) Z_{2} & =R_{2}\left(X_{2}, Y_{2}\right) Z_{2}-\left(\left\|g r a d_{1} f\right\|^{2}+\frac{\bar{r} f^{2}}{n(n-1)}\right)\left[g_{2}\left(Y_{2}, Z_{2}\right) X_{2}-g_{2}\left(X_{2}, Z_{2}\right) Y_{2}\right], \\
\mathcal{C}\left(X_{1}, Y_{2}\right) Z_{1} & =\frac{1}{f} H_{1}^{f}\left(X_{1}, Z_{1}\right) Y_{2}+\frac{\bar{r}}{n(n-1)} g_{1}\left(X_{1}, Z_{1}\right) Y_{2}, \\
\mathcal{C}\left(X_{1}, Y_{2}\right) Z_{2} & =-f g_{2}\left(Y_{2}, Z_{2}\right) \nabla_{X_{1}}^{1} \operatorname{grad}_{1} f-\frac{\bar{r} f^{2}}{n(n-1)} g_{2}\left(Y_{2}, Z_{2}\right) X_{1}, \\
\mathcal{C}\left(X_{1}, \partial_{t}\right) Z_{1} & =\frac{1}{h} H^{h}\left(X_{1}, Z_{1}\right) \partial_{t}+\frac{\bar{r}}{n(n-1)} g_{1}\left(X_{1}, Z_{1}\right) \partial_{t}, \\
\mathcal{C}\left(X_{2}, \partial_{t}\right) Z_{2} & =\frac{1}{h} H^{h}\left(X_{2}, Z_{2}\right) \partial_{t}+\frac{\bar{r} f^{2}}{n(n-1)} g_{2}\left(X_{2}, Z_{2}\right) \partial_{t}, \\
\mathcal{C}\left(X_{1}, \partial_{t}\right) Z_{2} & =\frac{1}{h} H^{h}\left(X_{1}, Z_{2}\right) \partial_{t}, \quad \mathcal{C}\left(X_{i}, \partial_{t}\right) \partial_{t}=h \bar{\nabla}_{X_{i}} g r a d h+\frac{\bar{r} h^{2}}{n(n-1)} X_{i}, \quad(i=1,2) .
\end{aligned}
$$

As a consequence of Proposition 4.2, we obtain:

Theorem 4.3. Let $\bar{M}=\left(M_{1} \times_{f} M_{2}\right) \times_{h} I$ be an $n\left(=m_{1}+m_{2}+1\right)$-dimensional sequential standard static spacetime endowed with the metric $\left.\bar{g}=g_{1} \oplus f^{2} g_{2}\right) \oplus h^{2}\left(-d t^{2}\right)$. Then, $(\bar{M}, \bar{g})$ is concircularly flat if and only if the following statements hold:

1. $\left(M_{1}, g_{1}\right)$ is of constant curvature $\kappa_{1}=\frac{\bar{r}}{n(n-1)}$.

2. $\left(M_{2}, g_{2}\right)$ is of constant curvature $\kappa_{2}=\frac{\bar{r} f^{2}}{n(n-1)}+\left\|g^{2 r a d} d_{1} f\right\|^{2}$.

3. $H_{1}^{f}+\frac{\bar{r} f}{n(n-1)} g_{1}=0$ and $H^{h}+\frac{\bar{r} h}{n(n-1)} g_{1}=0$ on $M_{1}$.

4. $H^{h}+\frac{\bar{r} h f^{2}}{n(n-1)} g_{2}=0$ on $M_{2}$. 
5. $H^{h}\left(X_{1}, Z_{2}\right)=0$, for all $X_{1} \in \chi\left(M_{1}\right)$ and $Z_{2} \in \chi\left(M_{2}\right)$.

Theorem 4.4. Let $\bar{M}=\left(M_{1} \times_{f} M_{2}\right) \times_{h} I$ be an $n\left(=m_{1}+m_{2}+1\right)$-dimensional sequential standard static spacetime endowed with the metric $\left.\bar{g}=g_{1} \oplus f^{2} g_{2}\right) \oplus h^{2}\left(-d t^{2}\right)$. Then, $(\bar{M}, \bar{g})$ is concircularly flat, then:

1. the warping function $h$ depends only on $M_{1}$,

2. the warping function $f$ satisfies the equation $X_{1}(\ln f)+\frac{\bar{r} h f^{2}}{n(n-1)}=0$,

3. $\left(M_{1}, g_{1}\right)$ is the conformal gradient soliton with potential function $h$.

Proof. Since the warping function $h$ is expressed as $h=h_{1}+h_{2}$, for each $h_{i} \in C^{\infty}\left(M_{i}\right),(i=1,2)$, from the last assertion of Theorem 4.3, we get $X_{1}(\ln f) \operatorname{grad} h_{2}=0$, which yields $h$ depends only on $M_{1}$. In this case, from the forth assertion of Theorem 4.3, we get (2). Also, from the third assertion of Theorem 4.3, similar condition with (3.2), which yields (3).

\subsection{Generalized Quasi Einsteinian Conditions on Sequential Warped Product Spacetimes}

In this section, we investigate generalized quasi Einstein condition on the sequential generalized RobertsonWalker spacetime. Of course, similar examination can be made on sequential standard static spacetimes. Since the results will be analogous, we will only present the results on the first mentioned class.

A Riemannian manifold $\left(\bar{M}^{n}, \bar{g}\right)(n>2)$ is called a generalized quasi Einstein manifold in the sense of Chaki [8], briefly $G(Q E)_{n}$, if its Ricci tensor of type $(0,2)$ is non-zero and satisfies the following condition

$$
\mathrm{Ric}=a \bar{g}+b A \otimes A+c[A \otimes B+B \otimes A],
$$

where $a, b, c$ are real valued, non-zero scalar functions on $\left(M^{n}, g\right)$ of which $b \neq 0, c \neq 0$ and $A$ and $B$ are two non-zero 1-forms such that they are dual of the orthonormal vector fields $U$ and $V$, respectively. That is,

$$
\bar{g}(U, \cdot)=A, \bar{g}(V, \cdot)=B, \bar{g}(U, V)=0 \text { and } \bar{g}(U, U)=\bar{g}(V, V)=1 .
$$

Note that, Catino [7] and Chaki [8] have introduced two different manifolds with different geometrical properties with the same name "generalized quasi Einstein". While the definition used in Subsection 3.1 is that of Catino, here we used the definition of Chaki.

In Lorentzian setting, the generalized quasi Einstein spacetime is a manifold whose Ricci tensor satisfies (4.5) and generators are given by (4.6), but in this case, $U$ is defined to be time-like, i.e., $\bar{g}(U, U)=-1$. From Notation 1-(v), these generator vector $U$ and $V$ can be also decomposed as:

$$
U=k \partial_{t}+U_{2}+U_{3}, \quad V=l \partial_{t}+V_{2}+V_{3}, \text { where } U_{i}, V_{i} \in \chi\left(M_{i}\right), i=2,3 \text { and } k, l \in \mathbb{R} .
$$

Let $\bar{M}=\left(I \times_{f} M_{2}\right) \times_{h} M_{3}$ be a $G(Q E)_{n}$ sequential generalized Robertson-Walker spacetime endowed with the metric $\bar{g}=\left(-\mathrm{d} t^{2} \oplus f^{2} g_{2}\right) \oplus h^{2} g_{3}$, and let $X_{i}, Y_{i} \in \chi\left(M_{i}\right)$, for $i=2,3$.

Computing both sides of (4.5) at $\partial_{t} \in \chi(I)$ and using Lemma 4.3 and equation (4.7), we obtain

$$
m_{2} \frac{\ddot{f}}{f}+\frac{m_{3}}{h} \frac{\partial^{2} h}{\partial t^{2}}=-a+b k^{2}+2 c k l .
$$

Similarly, computing both sides of (4.5) on $M_{2}$ and using Lemma 4.3 and equation (4.7), we obtain

$$
\mathrm{Ric}_{2}=\left(\mathrm{a}+\mathrm{f}^{\sharp}\right) \mathrm{g}_{2}+\frac{\mathrm{m}_{3}}{\mathrm{~h}} \mathrm{H}^{\mathrm{h}}+\mathrm{bA} \otimes \mathrm{A}+\mathrm{c}[\mathrm{A} \otimes \mathrm{B}+\mathrm{B} \otimes \mathrm{A}]
$$

and, computing both sides of (4.5) on $M_{3}$ and using Lemma 4.3 and equation (4.7), we obtain

$$
\mathrm{Ric}_{3}=\left(\mathrm{a}+\mathrm{h}^{\sharp}\right) \mathrm{g}_{3}+\mathrm{bA} \otimes \mathrm{A}+\mathrm{c}[\mathrm{A} \otimes \mathrm{B}+\mathrm{B} \otimes \mathrm{A}] .
$$

Since the generator $U$ is time-like and $U$ and $V$ are orthonormal, by assuming the conditions

$$
\left\{\begin{array}{l}
g_{2}\left(U_{2}, U_{2}\right)=\left(k^{2}+h^{2}-1\right) / f^{2}, \\
g_{2}\left(V_{2}, V_{2}\right)=\left(l^{2}-h^{2}+1\right) / f^{2}, \\
g_{2}\left(U_{2}, V_{2}\right)=k l / f^{2},
\end{array}\right.
$$


we arrive that the components $U_{3}$ and $V_{3}$ are orthonormal on $M_{3}$. Combining this result with (4.10), we reach the generalized quasi Einstein structure on $M_{3}$.

On the other hand, if we assume that $h$ is defined only on $M_{1}$, then we get $H^{h}=\operatorname{gradh}(\ln f) f^{2} g_{2}$. If we use this into (4.9) and assume that the conditions

$$
\left\{\begin{array}{l}
g_{3}\left(U_{3}, U_{3}\right)=\left(k^{2}-f^{2}-1\right) / h^{2}, \\
g_{3}\left(V_{3}, V_{3}\right)=\left(l^{2}-f^{2}+1\right) / h^{2}, \\
g_{3}\left(U_{3}, V_{3}\right)=k l / h^{2},
\end{array}\right.
$$

hold, then the components $U_{2}$ and $V_{2}$ become orthonormal on $M_{2}$. Combining this result with (4.10), we reach the generalized quasi Einstein structure on $M_{2}$.

Hence, we summarize these results as in follows:

Proposition 4.3. Let $\bar{M}=\left(I \times{ }_{f} M_{2}\right) \times_{h} M_{3}$ be a $G(Q E)_{n}$ sequential generalized Robertson-Walker spacetime endowed with the metric $\bar{g}=\left(-d t^{2} \oplus f^{2} g_{2}\right) \oplus h^{2} g_{3}$. Then:

1. the warping functions $f, h$ and the associated scalar functions $a, b, c$ are related by (4.8).

2. if the system (4.11) holds, then $\left(M_{3}, g_{3}\right)$ is a $G(Q E)_{m_{3}}$.

3. if the system (4.12) holds and $h$ is defined only on $M_{1}$, then $\left(M_{2}, g_{2}\right)$ is a $G(Q E)_{m_{2}}$.

We finish this work with some examples of the sequential generalized Robertson-Walker spacetime, which is also a $G(Q E)_{n}$.

Example 4.1. Let $\left(\mathbb{R}^{4}, \bar{g}\right)$ be a Lorentzian manifold endowed with the metric $\bar{g}$ given by

$$
d s^{2}=-(\mathrm{d} t)^{2}+t^{2}(\mathrm{~d} x)^{2}+\left(e^{2 t}\right)\left[(\mathrm{d} y)^{2}+(\mathrm{d} z)^{2}\right]
$$

where $\frac{1}{2}<t<1$ and $\left(x^{1}, x^{2}, x^{3}, x^{4}\right)=(t, x, y, z)$ are the standard coordinates of $\mathbb{R}^{4}$. Then, in [14], we proved that $\left(\mathbb{R}^{4}, \bar{g}\right)$ is a generalized quasi Einstein spacetime satisfying (4.5) with non zero and non constant scalar curvature $r=-2\left[\frac{2}{t}+3\right]$, whose associated scalar functions are defined by

$$
a=-2-\frac{1}{t}, \quad b=-2, \quad c=\frac{\sqrt{2 t-1}}{t}
$$

and the associated 1-forms are defined by

$$
\begin{gathered}
A_{i}= \begin{cases}t \sqrt{\frac{2 t-1}{2(1-t)}} & , \text { if } i=2 \\
0 & \text {,if } i=3,4 \\
\frac{-1}{\sqrt{2(1-t)}} & \text {,if } i=1\end{cases} \\
B_{i}= \begin{cases}\frac{t}{\sqrt{2(1-t)}} & \text {,if } i=2 \\
0 & \text {,if } i=3,4 \\
-\sqrt{\frac{2 t-1}{2(1-t)}} & , \text { if } i=1\end{cases}
\end{gathered}
$$

Moreover, the metric (4.13) is also a sequential generalized Robertson-Walker spacetime metric, whose warping functions $f(t)=t$ and $h(t)=e^{t}$.

Example 4.2. If we consider the spatially homogeneous and isotropic Bianchi type-V metric $\tilde{g}$ on $\mathbb{R}^{4}$ by

$$
d s^{2}=-(\mathrm{d} t)^{2}+t^{2}(\mathrm{~d} x)^{2}+e^{2(x+t)}\left[(\mathrm{d} y)^{2}+(\mathrm{d} z)^{2}\right]
$$

where $-1<t<1$ and $\left(x^{1}, x^{2}, x^{3}, x^{4}\right)=(x, y, z, t)$ are the standard coordinates of $\mathbb{R}^{4}$. Then the only non vanishing components of the Christoffel symbols, the curvature tensor and the Ricci tensor are

$$
\begin{array}{r}
\Gamma_{t x}^{x}=\frac{1}{t}, \quad \Gamma_{y y}^{x}=\Gamma_{z z}^{x}=-\frac{e^{2(x+t)}}{t^{2}}, \quad \Gamma_{x x}^{t}=t \\
\Gamma_{x y}^{y}=\Gamma_{x z}^{z}=\Gamma_{y t}^{y}=\Gamma_{z t}^{z}=1, \quad \Gamma_{y y}^{t}=\Gamma_{z z}^{t}=e^{2(x+t)},
\end{array}
$$




$$
\begin{gathered}
R_{x y y x}=R_{x z z x}=(1-t) e^{2(x+t)}, R_{y z z y}=\left(\frac{1}{t^{2}}-1\right) e^{4(x+t)}, \\
R_{y t t y}=R_{z t t z}=e^{2(x+t)}, R_{x y y t}=R_{x z z t}=\left(1-\frac{1}{t}\right) e^{2(x+t)}, \\
\operatorname{Ric}_{\mathrm{xx}}=2(1-\mathrm{t}), \quad \operatorname{Ric}_{\mathrm{tt}}=2, \operatorname{Ric}_{\mathrm{xt}}=2-\frac{2}{\mathrm{t}} \\
\operatorname{Ric}_{\mathrm{yy}}=\operatorname{Ric}_{\mathrm{zz}}=\left(\frac{2}{\mathrm{t}^{2}}-\frac{1}{\mathrm{t}}-2\right) \mathrm{e}^{3(\mathrm{x}+\mathrm{t})}
\end{gathered}
$$

and the components which can be obtained from these by symmetry properties. Also it can be shown that the scalar curvature is

$$
r=\frac{6}{t^{2}}-\frac{4}{t}-6
$$

which is non zero and non constant. Let us now define associated scalar functions as

$$
a=\frac{2}{t^{2}}-\frac{1}{t}-2, \quad b=\frac{2}{t^{2}}-2, \quad c=\left(\frac{2}{t^{2}}-\frac{2}{t}\right) \frac{1}{\cosh (2 t)}
$$

and the components of the associated 1 -forms as

$$
A_{i}=(t \sinh (t), 0,0,-\cosh (t))
$$

and

$$
B_{i}=\left(\frac{\cosh (t)}{t}, 0,0, \sinh (t)\right)
$$

where $i=\{1,2,3,4\}$ respectively. Then, under these constructions it can be shown that the generators are orthonormal such that the first one is time-like and $\tilde{g}$ satisfies (4.5). Thus, $\left(\mathbb{R}^{4}, \tilde{g}\right)$ is a a generalized quasi Einstein spacetime. Moreover, the metric (4.17) is also a sequential generalized Robertson-Walker spacetime metric, whose warping functions $f(t)=t$ and $h(t)=e^{(x+t)}$.

\section{Acknowledgments}

The author would like to express her sincere gratitude to reviewers for their valuable comments that helped to improve the quality of the manuscript.

\section{References}

[1] Ahsan, Z., Siddiqui, S. A. : Concircular curvature tensor and fluid spacetimes. Int. J. Theor. Phys. 48, 3202-3212 (2009).

[2] Allison, D. E.: Energy conditions in standard static space-times. Gen. Rel. Grav., 20, 115-122 (1988).

[3] Barros, A., Batista, R., Ribeiro Jr. E.: Rigidity of gradient almost Ricci solitons. Illinois J. Math. 56(4), 1267-1279 (2012).

[4] Beem, J. K., Ehrlich, P. E., Easley, K. L.: Global Lorentzian Geometry. (2nd Ed.), Marcel Dekker. New York (1996).

[5] Bishop, R. L., O'Neill, B.:Manifolds of negative curvature. Trans. Amer. Math. Soc. 145, 1-49 (1969).

[6] Blair, D.E., Kim, J.-S., Tripathi, M.M.: On the concircular curvature tensor of a contact metric manifold. J. Korean Math. Soc. 42(5), 883-892 (2005).

[7] Catino, G.: Generalized quasi Einstein manifolds with harmonic Weyl tensor. Math. Z. 271, 751-756 (2012).

[8] Chaki, M.C.: On Generalized quasi-Einstein manifold. Publ. Math. Debrecen. 58, 638-691 (2001).

[9] Cheeger, J., Colding, T. H.: Lower bounds on Ricci curvature and the almost rigidity of warped products. Ann. of Math. 144(2), 189-237 (1996).

[10] Chen, B. Y. : A simple characterization of generalized Robertson-Walker space-times. Gen. Relativ. Gravit. 46, 18-33 (2014).

[11] De, U. C., Shenawy, S., Ünal, B.: Sequential warped products: curvature and conformal vector fields. Filomat. 33(13), 4071-4083 (2019).

[12] De, U. C., Shenawy, S., Ünal, B.: Concircular Curvature on warped product manifolds and applications. Bull. Malays. Math. Sci. Soc. 43, 3395$3409(2020)$.

[13] Dobarro, F, Ünal, B. : Special standard static spacetimes. Nonlinear Analysis: Theory, Methods and Applications. 59(5), 759-770 (2004).

[14] Güler, S., Altay Demirbag, S. : A Study of generalized quasi Einstein spacetimes with applications in general relativity. Int. J. Theor. Phys. 55, 548-562 (2016).

[15] Güler, S.: On a class of gradient almost Ricci solitons. Bull. Malays. Math. Sci. Soc. 43, 3635-3650 (2020).

[16] Karaca, F., Özgür, C.: On quasi-Einstein sequential warped product manifolds. Journal of Geom. Phys. 165, 104248 (2021).

[17] Mantica, C. A, Molinari, L. G., De, U. C.: A condition for a perfect fluid space-time to be a generalized Robertson-Walker space-time. J. Math. Phys. $57(2), 022508(2016)$. 
[18] Mantica, C. A, Suh, Y. J., De, U. C. : A note on generalized Robertson-Walker space-times. Int. J. Geom. Meth. Mod. Phys. 13, 1650079 (2016).

[19] O'Neill, B.: Semi Riemannian Geometry with Applications to Relativity. Pure and Applied Ser. Academic Press. New York (1983).

[20] Shenawy, S.: A note on sequential warped product manifolds. Preprint arxiv:1506.06056v1 (2015).

[21] Souso, M. L., Pina, R.: Gradient Ricci solitons with structure of warped product. Results Math. 17, 825-840 (2017).

[22] Stephani, H., Kramer, D., MacCallum, M., Hoenselaers, C., Herlt, E.: Exact solutions of Einstein's field equations. Second Edition, Cambridge University Press. Cambridge (2003)

[23] Tashiro, Y.: Complete Riemannian manifolds and some vector fields. Trans. Amer. Math. Soc. 117, 251-275 (1965).

[24] Yano, K., Kon, M.: Structures on Manifolds. World Scientific Publishing. Singapore (1984).

[25] Yano, K.: Concircular geometry I. Concircular transformations. Proc. Imp. Acad. Tokyo. 16, 195-200 (1940).

[26] Yun, G., Co, J., Hwang, S. : Bach-flat h-almost gradient Ricci solitons. Pacific J. Math. 288(2), 475-488 (2017).

\section{Affiliations}

\section{SINEM GÜLER}

AdDRESS: Department of Industrial Engineering, Istanbul Sabahattin Zaim University, 34303, Halkall, Turkey. E-MAIL: sinem.guler@izu.edu.tr

ORCID ID:0000-0001-9994-2927 